OPEN ACCESS

Edited by:

Mami lima,

Kyoto University, Japan

Reviewed by:

Yajia Gu,

Fudan University, China Jeon Hor Chen,

University of California, Irvine,

United States

*Correspondence: Elizabeth J. Sutton

suttone@mskcc.org

Specialty section: This article was submitted to

Cancer Imaging and

Image-directed Interventions,

a section of the journal

Frontiers in Oncology

Received: 17 August 2020 Accepted: 11 December 2020 Published: 04 February 2021

Citation:

Zhang M, Sadinski M, Haddad D, Bae MS, Martinez D, Morris EA,

Gibbs $P$ and Sutton EJ (2021)

Background Parenchymal

Enhancement on Breast MRI as a

Prognostic Surrogate: Correlation With Breast Cancer Oncotype Dx Score.

Front. Oncol. 10:595820.

doi: 10.3389/fonc.2020.595820

\section{Background Parenchymal Enhancement on Breast MRI as a Prognostic Surrogate: Correlation With Breast Cancer Oncotype Dx Score}

\author{
Michelle Zhang ${ }^{1,2}$, Meredith Sadinski ${ }^{1}$, Dana Haddad ${ }^{1,3,4,5}$, Min Sun Bae ${ }^{1,6}$, \\ Danny Martinez ${ }^{1}$, Elizabeth A. Morris ${ }^{1}$, Peter Gibbs ${ }^{1}$ and Elizabeth J. Sutton ${ }^{1 *}$
}

\begin{abstract}
${ }^{1}$ Department of Radiology, Memorial Sloan Kettering Cancer Center, New York, NY, United States, ${ }^{2}$ Department of Radiology, McGill University, Montreal, QC, Canada, ${ }^{3}$ Department of Radiology, Montefiore, New York, NY, United States, ${ }^{4}$ Department of Radiology, Mediclinic Middle East, Dubai, United Arab Emirates, ${ }^{5}$ College of Medicine, Mohammed Bin Rashid University of Medicine and Health Sciences, Dubai, United Arab Emirates, ${ }^{6}$ Department of Radiology, Seoul National University Hospital, Seoul, South Korea
\end{abstract}

Purpose: Breast MRI background parenchymal enhancement (BPE) can potentially serve as a prognostic marker, by possible correlation with molecular subtype. Oncotype Dx, a gene assay, is a prognostic and predictive surrogate for tumor aggressiveness and treatment response. The purpose of this study was to investigate the association between contralateral non-tumor breast magnetic resonance imaging (MRI) background parenchymal enhancement and tumor oncotype score.

Methods: In this retrospective study, patients with ER+ and HER2- early stage invasive ductal carcinoma who underwent preoperative breast MRI, oncotype risk scoring, and breast conservation surgery from 2008-2010 were identified. After registration, BPE from the pre and three post-contrast phases was automatically extracted using a k-means clustering algorithm. Four metrics were calculated: initial enhancement (IE) relative to the pre-contrast signal, late enhancement, overall enhancement (OE), and area under the enhancement curve (AUC). Histogram analysis was performed to determine first order metrics which were compared to oncotype risk score groups using Mann-Whitney tests and Spearman rank correlation analysis.

Results: This study included 80 women (mean age $=51.1 \pm 10.3$ years); 46 women were categorized as low risk $(\leq 17)$ and 34 women were categorized as intermediate/high risk $(\geq 18)$ according to Oncotype Dx. For the mean of the top $10 \%$ pixels, significant differences were noted for IE ( $p=0.032), O E(p=0.049)$, and AUC $(p=0.044)$. Using the risk score as a continuous variable, correlation analysis revealed a weak but significant 
correlation with the mean of the top 10\% pixels for IE ( $r=0.26, p=0.02), \mathrm{OE}(r=0.25, p=$ $0.02)$, and $A \cup C(r=0.27, p=0.02)$.

Conclusion: BPE metrics of enhancement in the non-tumor breast are associated with tumor Oncotype Dx recurrence score, suggesting that the breast microenvironment may relate to likelihood of recurrence and magnitude of chemotherapy benefit.

Keywords: breast cancer, magnetic resonance imaging, oncotype, risk score, background parenchymal enhancement

\section{INTRODUCTION}

According to the Breast Imaging Reporting and Data System (BIRADS) guidelines, background parenchymal enhancement (BPE) should be routinely reported for breast magnetic resonance imaging (MRI). BPE can influence tumor detection on MRI and the sensitivity/specificity of the interpretation (1-3). However, there has been mounting evidence that BPE may also be of additional value. Theoretically, it can be a surrogate for the breast vascular microenvironment, with the potential to encourage or discourage tumor development, growth, and response to treatment. Higher $\mathrm{BPE}$ is associated with high risk patients (such as BRCA carriers) and premenopausal hormonal status (2). Recent preliminary studies suggest that BPE may serve as a prognostic marker, with findings supporting a possible correlation between BPE and molecular subtypes, particularly for distinguishing between luminal A and luminal B type cancers, and a possible relationship with recurrencefree survival (4-6). Although BPE is at times a relatively subjective BI-RADS descriptor, multiple papers have attempted to quantify BPE objectively, finding that it is positively correlated with breast cancer odds, even after adjusting for the amount of fibroglandular tissue (FGT) $(7,8)$.

Oncotype Dx (Genomic Health Inc., Redwood City, CA) is a validated 21-gene assay which is both prognostic and predictive. It provides the 10-year likelihood of breast cancer recurrence and helps to predict the likelihood of benefit from chemotherapy in patients diagnosed with early-stage estrogen receptor positive $(\mathrm{ER}+)$ and human epidermal growth factor receptor 2 negative (HER2-) breast cancer. The Oncotype Dx Recurrence Score (ODxRS) ranges from $0-100$ and is often subdivided into three risk categories: low $(\leq 17)$, intermediate (18-30) and high $(\geq 31)$. Clinically, a low ODxRS can potentially change patient management, because chemotherapy may not be recommended due to low benefits. Studies have validated the Oncotype Dx test in both node negative and positive patients $(9,10)$. Currently, ODxRS is being incorporated into breast cancer treatment guidelines (11).

The tumor microenvironment is a complex entity with both intrinsic (e.g., DNA abnormality) and extrinsic characteristics (e.g., oxygen tension and nutrients) (12). The enhancement of the breast is related to its vascularity, and it would be logical to hypothesize that the blood flow to the breast, which brings in the necessary nutrients, oxygen and other metabolites, is contributing to the metabolic environment with certain factors that could promote or discourage tumoral cellular growth. In fact, it has been shown that there is significant correlation between BPE and breast parenchymal metabolic activity as measured by 2-deoxy-2- [fluorine-18] fluoro-D-glucose positron emission tomography/ computed tomography (18F-FDG PET/CT) $(13,14)$. Thus, the increased metabolic activity of a breast with increased BPE could potentially provide a more favorable environment for tumoral growth and be a marker for breast cancer aggressiveness. In addition, BPE can be influenced by both endogenous and exogenous hormones; for example, it has been shown to be sensitive to the menstrual cycle fluctuations (15). Increase in hormonal stimulation such as during breastfeeding or ingestion of hormonal replacement therapy (HRT) has been shown to increase BPE, whereas hormonal suppression therapy such as aromatase inhibitors and Tamoxifen has been shown to decrease BPE $(15,16)$. By extension, as hormonal variability confers changes to the BPE, it could also in turn confer breast cancer risk stratification.

On MRI, a patient's breasts usually demonstrate symmetric parenchymal enhancement with similar kinetic curves throughout the contrast-enhanced time course, consistent with their having similar microenvironments. Because of this, we hypothesize that in women with breast cancer, the contralateral healthy breast represents the microenvironment from which the malignancy developed and could be predictive of tumor aggressiveness. The purpose of this study was to investigate if there is a relationship between breast cancer ODxRS and BPE in the contralateral healthy breast. This may elucidate the influence of BPE on tumor behavior and response to treatment using an objective metric measurement of BPE.

\section{MATERIALS AND METHODS}

\section{Patients}

The institutional review board approved this Health Insurance Portability and Accountability Act-compliant retrospective study and waived written informed consent. Between 2008 and 2010, we identified 80 consecutive breast cancer patients with ER+ and HER2 - early stage invasive ductal carcinoma who underwent preoperative breast MRI, Oncotype Dx and breast conservation surgery at our institution. Early stage cancer was defined as TMN staging 1 or 2. Patients with a prior history of breast cancer were excluded. All 80/80 patients overlap with the cohort used by Sutton et al. in 2015 (17). The prior study evaluated the associated between Oncotype Dx and the morphologic and texture-based image features of the cancer on MRI, whereas here we investigated the association between the contralateral 
non-tumor breast MRI FGT enhancement and tumor oncotype score.

\section{Imaging Protocol}

Breast MRI was performed using a protocol which included a precontrast T1-weighted sequence and three T1-weighted post-contrast phases. All images were acquired with a $1.5 \mathrm{~T}(\mathrm{n}=47 ; 59 \%)$ or $3.0 \mathrm{~T}$ ( $\mathrm{n}=33 ; 41 \%$ ) MRI system (Signa or Signa HDX; GE Medical Systems, Waukesha, WI). A dedicated 8 channel breast coil was used in all patients. Sagittal T1-weighted fat-suppressed 2D multi-slice acquisitions were acquired before and sequentially three times after intravenous administration of $0.1 \mathrm{mmol}$ gadopentetate dimeglumine per kilogram body weight (Magnevist; Berlex Laboratories/Bayer Health Care Pharmaceuticals, Montville, NJ) at a rate of $2 \mathrm{ml} / \mathrm{sec}$ with an automatic injector (Medrad, Warrendale, PA) and a 20-second scan delay using the following parameters: repetition time (TR, sec)/ echo time (TE, sec), 6.81 (5.08-11.30)/4.20 (1.95-4.20); flip angle $\left(^{\circ}\right)$, 10 (10-12), acquisition matrix, 256×192 (256-320×160-256); inplane resolution (mm), $0.78 \times 0.78(0.39-0.94)$; slice thickness $(\mathrm{mm}), 3$ (3-3); and temporal resolution $\sim 90$ secs.

\section{Imaging Analysis}

BPE of the contralateral non-cancerous breast was analyzed on a pixel-by-pixel basis. The fibroglandular tissue was segmented semiautomatically as follows using custom software written in MATLAB (Mathworks, Natick, NA). A radiologist (MZ, radiologist with breast imaging fellowship) first drew a line segment separating the chest wall from the breast tissue on orthogonal sagittal and axial maximum intensity projections of the first post-contrast T1-weighted image. A Gaussian smoothing filter was then applied, and the breast tissue was automatically thresholded from the background. An erosion filter was applied to the resulting segmentation to compensate for overestimation due to the smoothing step and to exclude skin. A rigid, intensity-based registration was applied to the pre- and post-contrast images to account for patient motion during the exam. The breast segmentation was then propagated to the pre-contrast T1weighted image and the fibroglandular tissue was extracted using $\mathrm{k}$-means clustering on the pre-contrast T1-weighted image. Finally, image registration and segmentation were checked by another radiologist ( $\mathrm{DH}$, radiologist with breast imaging fellowship), with manually adjustment applied as necessary.

Initial, overall, and late enhancement were calculated using the pre-contrast $\mathrm{T} 1$-weighted signal as the baseline. Initial enhancement (IE) was calculated as the percentage increase in signal from the pre-contrast (baseline) image to the first postcontrast image with respect to the baseline signal. Similarly, overall enhancement (OE) was calculated as the percentage increase in signal from baseline to the final post-contrast image with respect to the baseline signal. Late enhancement was evaluated as the percentage increase in signal from the first post contrast image to the last post contrast image with respect to the first post contrast image. Finally, the area under the enhancement curve (AUC) for total contrast enhancement was calculated, normalized to the baseline signal intensity (Figure 1).

All pixels with negative signal changes post-contrast injection were removed, since this indicates that no enhancement has occurred, and any signal fluctuations noted are due to signal to noise effects. Similarly, pixels with enhancement over 300\% were removed and regarded as demonstrating non-physiologically acceptable BPE. Such high percentage enhancement rates can be attributed to low pre-contrast signal followed by normal signal to noise-based fluctuations in the signal recorded during the dynamic time course.

Objective BPE calculations were presented as median and interquartile ranges. Since negative values of initial and total enhancement are precluded by definition, the pixel-by-pixel histogram of enhancement for each individual case could be considered as non-normally distributed. Therefore, median values and percentiles were used as summary variables for the central tendency and spread of the data. Reflecting previous work by van der Velden et al., the mean of the top $10 \%$ of pixels on the enhancement histograms was also calculated for each case, the decision of which was determined a priori (18). Focusing on the most enhancing pixels in breast lesions using similar techniques such as hot spot analysis has been shown to provide greater discrimination to predict response to neoadjuvant chemotherapy among patients with breast cancer (19).

\section{Tissue Testing}

All breast cancers underwent the standard Oncotype DX test to derive the Oncotype Dx recurrence score (ODxRS). Using ODxRS, patients were dichotomized into a low risk group (those with scores $\leq 17$ ) and an intermediate/high risk group (those with scores $\geq 18$ ). This was deemed appropriate due to the dichotomy of clinical management based on risk stratification by ODxRS; "low risk" patients would potentially not be offered additional chemotherapy (due to low benefit) and the "intermediate/high risk patients" would potentially be offered more aggressive therapies due to higher predicted recurrence and thus higher benefit of chemotherapy.

\section{Statistical Analysis}

Patient and clinical characteristics were compared between patients in the low and intermediate/high groups, using Fisher's exact test or the chi-square test where appropriate. All analyses were performed using MATLAB (v 9.1.0 R2016b MathWorks, Natick, MA). After appropriate dichotomization comparison of low and intermediate/ high risk group, first order statistics derived from the four enhancement curve parameters was performed using the independent sample Mann-Whitney $U$ test, with a $\mathrm{p}$-value $<0.05$ regarded as indicating a significant difference between groups. The Spearman rank correlation analysis was used to assess the relationships between the four enhancement curve parameters and the continuous ODxRS metric, with a p-value $<0.05$ indicating the presence of a significant correlation between parameters.

\section{RESULTS}

\section{Patients}

The mean age of the study population was 51.2 years (range, 27.4-77.6). Table 1 shows the patient characteristics. All patients underwent breast conserving surgery. The median ODxRS in the 

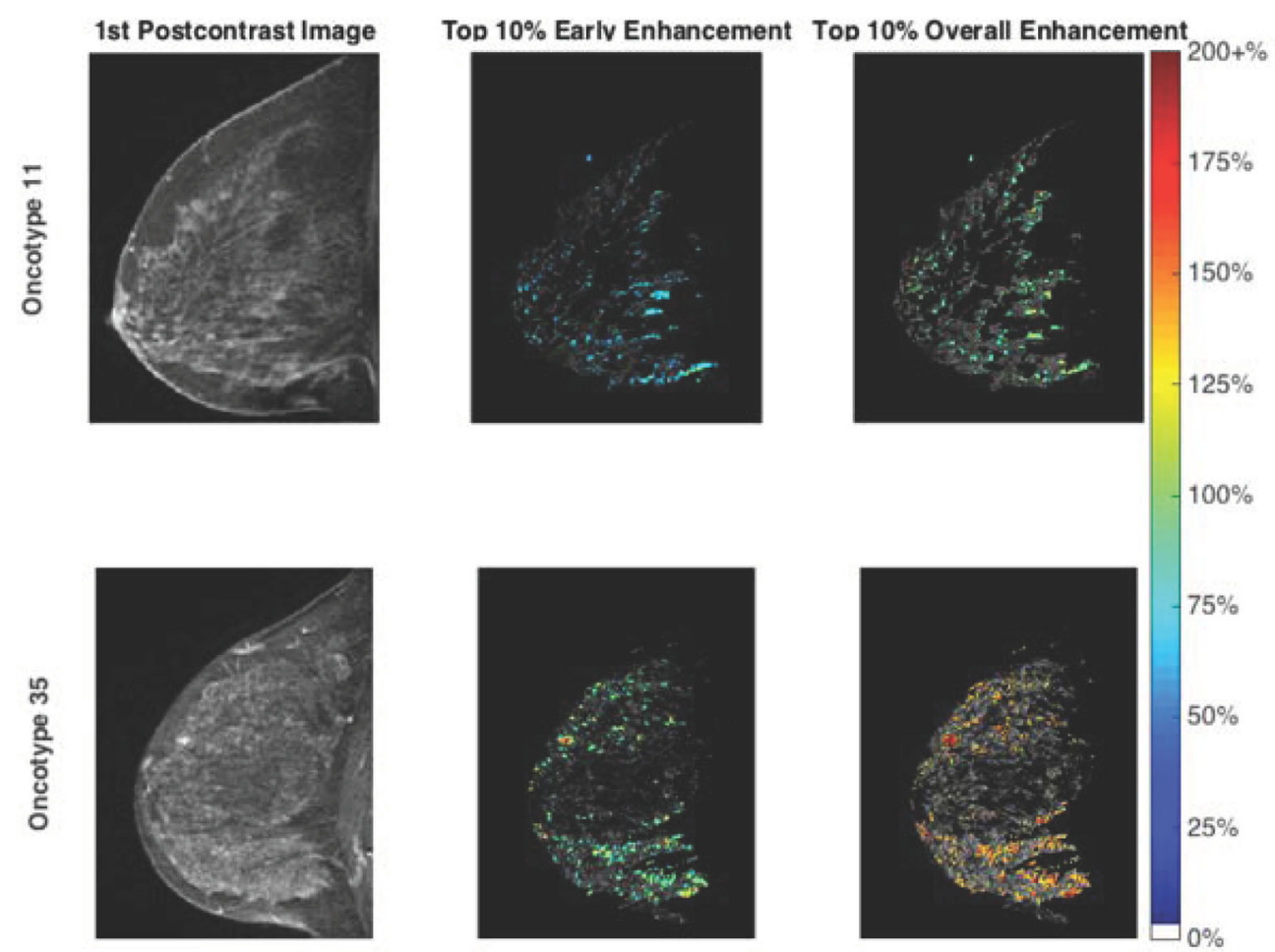

FIGURE 1 | Fibroglandular tissue enhancement in a low risk group patient (top row) and a high risk group patient (bottom row). The first postcontrast images are illustrated (left) alongside segmented fibroglandular tissue with early enhancement overlay (middle) and overall enhancement overlay (right). Note the higher enhancement values evident for the high risk group patient. Oncotype Dx is a prognostic and predictive surrogate for tumor aggressiveness and treatment response. A Oncotype Dx score of 11 is a representative example of a patient that is a low risk. Oncotype Dx score of 35 is a representative example of a patient that is at intermediate/high risk. The "top 10\%" corresponds to the voxels exhibiting the 10\% highest enhancement level in the middle and right figures.

study population was 16.5 (range: 0-78). Based on OdxRS, 46 (58\%) patients were categorized into the low risk group (0-17) and $34(42 \%)$ patients were categorized into the intermediate/ high risk groups $(\geq 18)$. From Table $\mathbf{1}$, it can be seen that patients in the intermediate/high risk group were more likely to be PR negative ( $p=0.028)$, HER2 positive ( $p=0.029)$, have a higher nuclear grade $(\mathrm{p}=0.030)$, and also less likely to be receiving hormone therapy $(\mathrm{p}=0.004)$, compared with the low risk group. No significant difference in overall BPE score was noted between the two groups $(\mathrm{p}=0.642)$.

\section{Relationship Between the Four Enhancement Curve Parameters and ODxRS Categories}

Borderline significant differences were noted between the two groups using the Mann-Whitney $U$ test for median values of IE $(\mathrm{p}=0.07)$ and $\mathrm{OE}(\mathrm{p}=0.09)$. When mean values of the top $10 \%$ of pixels were considered, significant differences were noted for IE $(p=0.03), O E(p=0.049)$ and AUC $(p=0.04)$. Greater IE, OE, and AUC were noted for the intermediate/high risk group compared to the low risk group (Table 2). Figure 1 illustrates the first postcontrast images alongside segmented FGT with higher enhancement values for a high risk group patient compared with a low risk group patient.

\section{Relationship Between the Four Enhancement Curve Parameters and ODxRS as a Continuous Variable}

Using ODxRS as a continuous variable, Spearman rank correlation analysis revealed weak but significant positive correlations between ODxRS and the mean of the top $10 \%$ of pixels for IE $(\mathrm{r}=0.26, \mathrm{p}=$ $0.02)$, OE $(r=0.25, p=0.02)$, and AUC ( $r=0.27, p=0.02)$ (Figure 2). No significant correlations were noted when utilizing median values (Table 3). With a sample size of 80 and employing a type I error rate of 0.05 , then for a correlation of $\sim 0.25$ a type II error rate of 0.35 (65\% power) can be expected.

\section{DISCUSSION}

BPE in the contralateral non-tumor breast positively correlates with ODxRS. This suggests that the microenvironment of the breast may predict tumor biology including the likelihood of recurrence and the magnitude of chemotherapy benefit. Given that we used the quantitative metric measurement of BPE rather than the subjective categorization by the interpreting radiologist, the relationship is more objective and reproducible. The correlation is more significant when considering the top $10 \%$ $\mathrm{BPE}$ and after dichotomizing ODxRS. 
TABLE 1 | Patient Characteristics.

\begin{tabular}{|c|c|c|c|}
\hline Categorical variable & $\begin{array}{l}\text { Low Risk } \\
(n=46)\end{array}$ & $\begin{array}{l}\text { Intermediate/High Risk } \\
\qquad(\mathrm{n}=34)\end{array}$ & p-value \\
\hline ER status & & & 0.073 \\
\hline Negative & $0(0 \%)$ & $3(9 \%)$ & \\
\hline Positive & $46(100 \%)$ & 31 (91\%) & \\
\hline PR status & & & 0.028 \\
\hline Negative & $2(4 \%)$ & $7(21 \%)$ & \\
\hline Positive & 44 (96\%) & 27 (79\%) & \\
\hline HER2 status & & & 0.029 \\
\hline Negative & 46 (100\%) & 30 (88\%) & \\
\hline Positive & $0(0 \%)$ & $4(12 \%)$ & \\
\hline Nodal status & & & 0.464 \\
\hline Negative & 42 (90\%) & 30 (88\%) & \\
\hline Positive & $4(10 \%)$ & $4(12 \%)$ & \\
\hline Histology grade & & & 0.216 \\
\hline 1 & 4 (9\%) & $1(3 \%)$ & \\
\hline 2 & 18 (39\%) & $9(26 \%)$ & \\
\hline 3 & 24 (52\%) & 24 (71\%) & \\
\hline Nuclear grade & & & 0.030 \\
\hline 1 & 4 (9\%) & $0(0 \%)$ & \\
\hline 2 & $29(63 \%)$ & $14(41 \%)$ & \\
\hline 3 & $10(22 \%)$ & $14(41 \%)$ & \\
\hline Unknown & $3(6 \%)$ & $6(18 \%)$ & \\
\hline Family history & & & 0.192 \\
\hline No & 26 (57\%) & $15(44 \%)$ & \\
\hline Yes & 20 (43\%) & $19(56 \%)$ & \\
\hline Menopausal status & & & 0.207 \\
\hline Pre-menopausal & $31(67 \%)$ & $19(56 \%)$ & \\
\hline Post-menopausal & 15 (33\%) & $15(44 \%)$ & \\
\hline HRT & & & 0.106 \\
\hline No & 43 (93\%) & 27 (79\%) & \\
\hline Yes & $3(7 \%)$ & $6(18 \%)$ & \\
\hline Unknown & $0(0 \%)$ & $1(3 \%)$ & \\
\hline Hormone therapy & & & 0.004 \\
\hline No & $0(0 \%)$ & $6(18 \%)$ & \\
\hline Yes & 46 (100\%) & $28(82 \%)$ & \\
\hline Radiation therapy & & & 0.203 \\
\hline No & $3(7 \%)$ & $5(15 \%)$ & \\
\hline Yes & 43 (93\%) & 29 (85\%) & \\
\hline BPE & & & 0.642 \\
\hline Minimal & $8(17 \%)$ & $7(21 \%)$ & \\
\hline Mild & 17 (37\%) & 12 (35\%) & \\
\hline Moderate & 15 (33\%) & $7(21 \%)$ & \\
\hline Marked & $6(13 \%)$ & $8(23 \%)$ & \\
\hline
\end{tabular}

HRT, hormone replacement therapy.

TABLE 2 | Top 10\% BPE correlating with dichotomized Oncotype Dx score. Values are given as median (range).

\begin{tabular}{lcccc}
\hline & Initial Enh (\%) & Overall Enh (\%) & Late Enh (\%) & AUC \\
\hline Group 1 & 53.1 & 72.6 & 26.5 & 2.71 \\
$(\boldsymbol{n}=\mathbf{4 6})$ & $(20.2$ to 129.2) & $(29.9$ to 185.0$)$ & $(13.2$ to 84.2$)$ & $(1.10$ to 6.79$)$ \\
Group 2/3 & 63.9 & 84.5 & 28.9 & 3.23 \\
$(\boldsymbol{n}=\mathbf{3 4 )}$ & $(30.3$ to 159.1) & $(53.9$ to 195.0$)$ & $(17.9$ to 50.5$)$ & $(1.84$ to 7.57$)$ \\
$\boldsymbol{P}$-value & 0.03 & 0.049 & 0.45 & 0.04 \\
\hline
\end{tabular}

Enh, Enhancement; AUC, Area under the enhancement curve.

Group 1 = low risk oncotype score group (ODxRS $\leq 17)$.

Group 2/3 = intermediate/high risk oncotype score group (ODxRS $\geq 18$ ).

The decision to use the mean of the top $10 \%$ of the BPE was determined a priori. This is similar to hot spot analysis and was previously proposed by other papers $(18,20)$. Given that we hypothesized that the $\mathrm{BPE}$ represents the microenvironment from which the tumor arose, the top enhancing portion of the breast is mostly likely the most hormonally active, or susceptible. While there may be great heterogeneity across the breast in terms of BPE, focusing on the top $10 \%$ would ensure honing in on the most vascularized and sensitive part of the breast. This was affirmed by our study which showed a positive correlation between tumor aggressiveness (ODxRS) and the top 10\% BPE. In addition, in our study, there was no spatial localization of the top $10 \%$ most enhancing parenchyma and the pixels were equally likely to be scattered disparately throughout the breast; therefore, we hypothesize that the risk assessment conveyed via BPE would be globally for the breast itself and not for a particular region of the breast.

Interestingly, the correlation between ODxRS and BPE was stronger when the scores are dichotomized rather than when used as a continuous variable. Clinically speaking, low-risk patients are usually not recommended to receive adjuvant chemotherapy whereas intermediate/high-risk patients are recommended to receive additional chemotherapy (21). Thus, this dichotomization would make sense as it would change clinical management. There is substantial benefit gained from additional adjuvant therapy for the high risk group. Management for patients in the intermediate group is somewhat controversial with most patients being offered chemotherapy but the recently published prospective TAILORx trial suggests that endocrine therapy may be non-inferior to chemoendocrine therapy in particular subsets of patients (e.g., older women above 50 years of age with ODxRS score of 25 or lower); however, more validation studies would be needed to better define these subgroups (21-23).

The current literature on the association of BPE with tumor ODxRS is scarce. However, there is growing evidence that the microenvironment of the breast, as evidenced by the BPE, is associated with different tumoral behavior and can provide prognostic factors. It has been shown that BPE in the contralateral breast in breast cancer patients, especially the top $10 \%$ parenchymal enhancement as our study has similarly assessed, is associated with overall long-term patient outcome (20). In a similar fashion, increased BPE on pre-treatment MRI for patients undergoing neoadjuvant chemotherapy has been shown to be significantly correlated with worse recurrence-free survival (24). Remarkably, a parallel study looking instead at the BPE around ductal carcinoma in situ in the cancerous breast showed that the increased signal enhancement ratio in the ipsilateral cancerous breast around the tumor was associated with worse ipsilateral tumor recurrence-free survival (25).

Our study affirms that breast BPE can serve as a surrogate for the microenvironment that predisposes carcinogenesis, in line with previous studies. It can potentially help predict treatment response and disease recurrence and triage patients into different risk stratification groups. Ultimately, BPE can help to identify tumoral characteristics that would indicate more aggressive behavior and help tailor treatment and clinical decision making. If this association is further validated, additional studies could be performed to evaluate whether this risk would also be conferred in non-cancerous screening patients, in order to help prevent cancer 


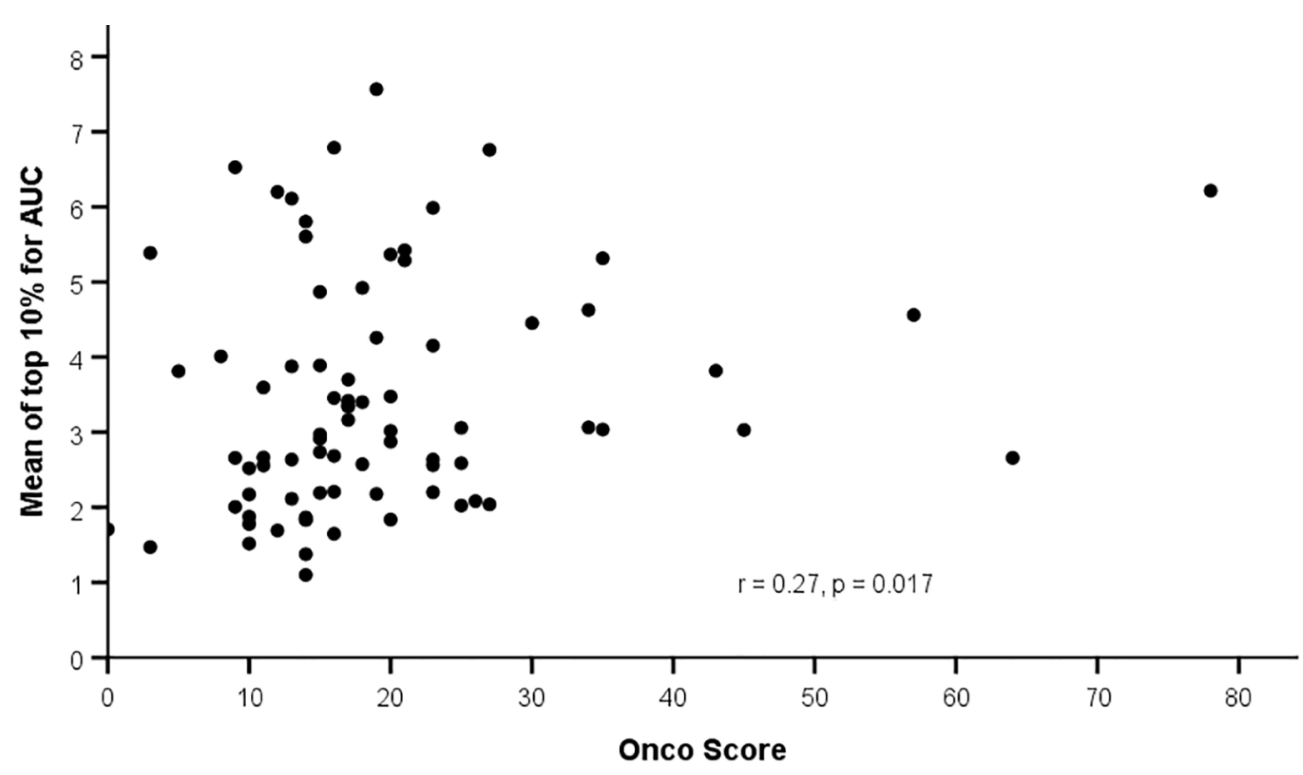

FIGURE 2 | Correlation between background parenchymal enhancement (mean top 10\%) and Oncotype Dx Recurrence Score.

TABLE 3 | Correlation analysis of BPE metrics with continuous Oncotype Dx score.

\begin{tabular}{lcc}
\hline & Spearman rank correlation coefficient & $\boldsymbol{P}$-value \\
\hline $\begin{array}{ll}\text { Median } \\
\text { Initial enhancement }\end{array}$ & 0.07 \\
Overall enhancement & 0.20 & 0.05 \\
Late enhancement & 0.22 & 0.13 \\
AUC & 0.17 & 0.06 \\
Top 10\% of pixels & 0.21 & \\
Initial enhancement & & 0.02 \\
Overall enhancement & 0.26 & 0.02 \\
Late enhancement & 0.25 & 0.22 \\
AUC & 0.14 & 0.02 \\
\hline
\end{tabular}

BPE, Background parenchymal enhancement; AUC, Area under the enhancement curve.

development rather than only passively treating the tumor once it has already developed.

Our study is limited by the fact that it is a retrospective study from a single institution and the sample size was small. In addition, there may also be selection bias as only patients who were stratified by the Oncotype Dx test were included for analysis. Conclusions are limited to the subset of ER+, HER2- breast cancer, and not generalizable to the broader patient population.

In conclusion, increased fibroglandular BPE on MRI in the contralateral non-tumor breast is correlated with higher Oncotype Dx scores, suggesting that BPE may be an imaging phenotype of the microenvironment that correlates with tumor aggressiveness and response to chemotherapy. Future studies with a larger patient sample size and treatment response followup may help further elucidate this association. Fibroglandular $\mathrm{BPE}$ is a very promising predictive factor as an additional tool for risk stratification and treatment management in patients with breast cancer.

\section{DATA AVAILABILITY STATEMENT}

The data analyzed in this study is subject to the following licenses/ restrictions. Data will be shared with qualified researchers whose proposed use of the data has been approved. Requests to access these datasets should be directed to ES (suttone@mskcc.org).

\section{ETHICS STATEMENT}

The studies involving human participants were reviewed and approved by Institutional Review Board, Memorial Sloan Kettering Cancer Center. Written informed consent for participation was not required for this study in accordance with the national legislation and the institutional requirements.

\section{AUTHOR CONTRIBUTIONS}

All authors contributed to the article and approved the submitted version.

\section{FUNDING}

This research was funded in part through the NIH/NCI Cancer Center Support Grant P30 CA008748, the Breast Cancer Research Foundation, and the Susan G. Komen Foundation.

\section{ACKNOWLEDGMENTS}

The authors thank Joanne Chin, MFA, ELS, for her help in editing this manuscript. 


\section{REFERENCES}

1. Giess CS, Raza S, Birdwell RL. Patterns of nonmasslike enhancement at screening breast MR imaging of high-risk premenopausal women. Radiographics Rev Publ Radiol Soc North America Inc (2013) 33(5):134360. doi: $10.1148 / \mathrm{rg} .335125185$

2. Lewin AA, Kim SG, Babb JS, Melsaether AN, McKellop J, Moccaldi M, et al. Assessment of Background Parenchymal Enhancement and Lesion Kinetics in Breast MRI of BRCA 1/2 Mutation Carriers Compared to Matched Controls Using Quantitative Kinetic Analysis. Acad Radiol (2016) 23 (3):358-67. doi: 10.1016/j.acra.2015.11.011

3. Telegrafo M, Rella L, Stabile Ianora AA, Angelelli G, Moschetta M. Effect of background parenchymal enhancement on breast cancer detection with magnetic resonance imaging. Diagn Interventional Imaging (2016) 97 (3):315-20. doi: 10.1016/j.diii.2015.12.006

4. Wu J, Sun X, Wang J, Cui Y, Kato F, Shirato H, et al. Identifying relations between imaging phenotypes and molecular subtypes of breast cancer: Model discovery and external validation. J Magnetic Resonance Imaging JMRI (2017) 46(4):1017-27. doi: 10.1002/jmri.25661

5. Mazurowski MA, Zhang J, Grimm LJ, Yoon SC, Silber JI. Radiogenomic analysis of breast cancer: luminal B molecular subtype is associated with enhancement dynamics at MR imaging. Radiology (2014) 273(2):365-72. doi: 10.1148/radiol.14132641

6. Lim Y, Ko ES, Han BK, Ko EY, Choi JS, Lee JE, et al. Background parenchymal enhancement on breast MRI: association with recurrence-free survival in patients with newly diagnosed invasive breast cancer. Breast Cancer Res Treat (2017) 163(3):573-86. doi: 10.1007/s10549-017-4217-5

7. Wu S, Zuley ML, Berg WA, Kurland BF, Jankowitz RC, Sumkin JH, et al. DCE-MRI Background Parenchymal Enhancement Quantified from an Early versus Delayed Post-contrast Sequence: Association with Breast Cancer Presence. Sci Rep (2017) 7(1):2115. doi: 10.1038/s41598-017-02341-8

8. King V, Brooks JD, Bernstein JL, Reiner AS, Pike MC, Morris EA. Background parenchymal enhancement at breast MR imaging and breast cancer risk. Radiology (2011) 260(1):50-60. doi: 10.1148/radiol.11102156

9. Albain KS, Barlow WE, Shak S, Hortobagyi GN, Livingston RB, Yeh IT, et al. Prognostic and predictive value of the 21-gene recurrence score assay in postmenopausal women with node-positive, oestrogen-receptor-positive breast cancer on chemotherapy: a retrospective analysis of a randomised trial. Lancet Oncol (2010) 11(1):55-65. doi: 10.1016/s1470-2045(09)70314-6

10. Paik S, Tang G, Shak S, Kim C, Baker J, Kim W, et al. Gene expression and benefit of chemotherapy in women with node-negative, estrogen receptor-positive breast cancer. J Clin Oncol (2006) 24(23):3726-34. doi: 10.1200/jco.2005.04.7985

11. Harris L, Fritsche H, Mennel R, Norton L, Ravdin P, Taube S, et al. American Society of Clinical Oncology 2007 update of recommendations for the use of tumor markers in breast cancer. J Clin Oncol (2007) 25(33):5287-312. doi: $10.1200 / j c 0.2007 .14 .2364$

12. Lyssiotis CA, Kimmelman AC. Metabolic Interactions in the Tumor Microenvironment. Trends Cell Biol (2017) 27(11):863-75. doi: 10.1016/ j.tcb.2017.06.003

13. Mema E, Mango VL, Guo X, Karcich J, Yeh R, Wynn RT, et al. Does breast MRI background parenchymal enhancement indicate metabolic activity? Qualitative and 3D quantitative computer imaging analysis. J Magnetic Resonance Imaging JMRI (2018) 47(3):753-9. doi: 10.1002/jmri.25798

14. Leithner D, Baltzer PA, Magometschnigg HF, Wengert GJ, Karanikas G, Helbich TH, et al. Quantitative Assessment of Breast Parenchymal Uptake on 18F-FDG PET/CT: Correlation with Age, Background Parenchymal Enhancement, and Amount of Fibroglandular Tissue on MRI. J Nuclear Med Off Publication Soc Nuclear Med (2016) 57(10):1518-22. doi: 10.2967/jnumed.116.174904

15. Heller SL, Young Lin LL, Melsaether AN, Moy L, Gao Y. Hormonal Effects on Breast Density, Fibroglandular Tissue, and Background Parenchymal
Enhancement. Radiographics Rev Publ Radiol Soc North America Inc (2018) 38(4):983-96. doi: 10.1148/rg.2018180035

16. Arslan G, Çelik L, Çubuk R, Çelik L, Atasoy MM. Background parenchymal enhancement: is it just an innocent effect of estrogen on the breast? Diagn Interventional Radiol (Ankara Turkey) (2017) 23(6):414-9. doi: 10.5152/ dir.2017.17048

17. Sutton EJ, Oh JH, Dashevsky BZ, Veeraraghavan H, Apte AP, Thakur SB, et al. Breast cancer subtype intertumor heterogeneity: MRI-based features predict results of a genomic assay. J Magnetic Resonance Imaging JMRI (2015) 42 (5):1398-406. doi: 10.1002/jmri.24890

18. van der Velden BHM, Elias SG, Bismeijer T, Loo CE, Viergever MA, Wessels LFA, et al. Complementary Value of Contralateral Parenchymal Enhancement on DCE-MRI to Prognostic Models and Molecular Assays in High-risk ER(+)/ HER2(-) Breast Cancer. Clin Cancer Res (2017) 23(21):6505-15. doi: 10.1158/ 1078-0432.ccr-17-0176

19. Pickles MD, Lowry M, Manton DJ, Gibbs P, Turnbull LW. Role of dynamic contrast enhanced MRI in monitoring early response of locally advanced breast cancer to neoadjuvant chemotherapy. Breast Cancer Res Treat (2005) 91(1):1-10. doi: 10.1007/s10549-004-5819-2

20. van der Velden BH, Dmitriev I, Loo CE, Pijnappel RM, Gilhuijs KG. Association between Parenchymal Enhancement of the Contralateral Breast in Dynamic Contrast-enhanced MR Imaging and Outcome of Patients with Unilateral Invasive Breast Cancer. Radiology (2015) 276(3):675-85. doi: 10.1148/radiol.15142192

21. McVeigh TP, Kerin MJ. Clinical use of the Oncotype DX genomic test to guide treatment decisions for patients with invasive breast cancer. Breast Cancer (Dove Med Press) (2017) 9:393-400. doi: 10.2147/bctt.s109847

22. Loncaster J, Armstrong A, Howell S, Wilson G, Welch R, Chittalia A, et al. Impact of Oncotype DX breast Recurrence Score testing on adjuvant chemotherapy use in early breast cancer: Real world experience in Greater Manchester, UK. Eur J Surg Oncol J Eur Soc Surg Oncol Br Assoc Surg Oncol (2017) 43(5):931-7. doi: 10.1016/j.ejso.2016.12.010

23. Sparano JA, Gray RJ, Makower DF, Pritchard KI, Albain KS, Hayes DF, et al. Adjuvant Chemotherapy Guided by a 21-Gene Expression Assay in Breast Cancer. New Engl J Med (2018) 379(2):111-21. doi: 10.1056/NEJMoa1804710

24. Choi JS, Ko ES, Ko EY, Han BK, Nam SJ. Background Parenchymal Enhancement on Preoperative Magnetic Resonance Imaging: Association With Recurrence-Free Survival in Breast Cancer Patients Treated With Neoadjuvant Chemotherapy. Medicine (2016) 95(9):e3000. doi: 10.1097/ md.0000000000003000

25. Kim SA, Cho N, Ryu EB, Seo M, Bae MS, Chang JM, et al. Background parenchymal signal enhancement ratio at preoperative MR imaging: association with subsequent local recurrence in patients with ductal carcinoma in situ after breast conservation surgery. Radiology (2014) 270 (3):699-707. doi: 10.1148/radiol.13130459

Conflict of Interest: EM received a grant from GRAIL Inc. for research not related to the present article. MS is currently employed by Promaxo in San Francisco, CA.

The remaining authors declare that the research was conducted in the absence of any commercial or financial relationships that could be construed as a potential conflict of interest.

Copyright (c) 2021 Zhang, Sadinski, Haddad, Bae, Martinez, Morris, Gibbs and Sutton. This is an open-access article distributed under the terms of the Creative Commons Attribution License (CC BY). The use, distribution or reproduction in other forums is permitted, provided the original author(s) and the copyright owner(s) are credited and that the original publication in this journal is cited, in accordance with accepted academic practice. No use, distribution or reproduction is permitted which does not comply with these terms. 